\title{
Supporting Information: Nanoconfinement Raises the Barrier to Hydrogen Atom Exchange between Water and Glucose
}

Samantha L. Miller, Benjamin P. Wiebenga-Sanford, Christopher D. Rithner, and Nancy E.

Levinger*

Department of Chemistry, Colorado State University, Fort Collins CO 80523-1872 USA

\section{Supporting Information:}

To validate the temperature of samples measured in EXSY NMR experiments, we measured the temperature of the equilibrated NMR probe before and after spectral collection using a methanol thermometer. We include a sample trace in Figure S1.

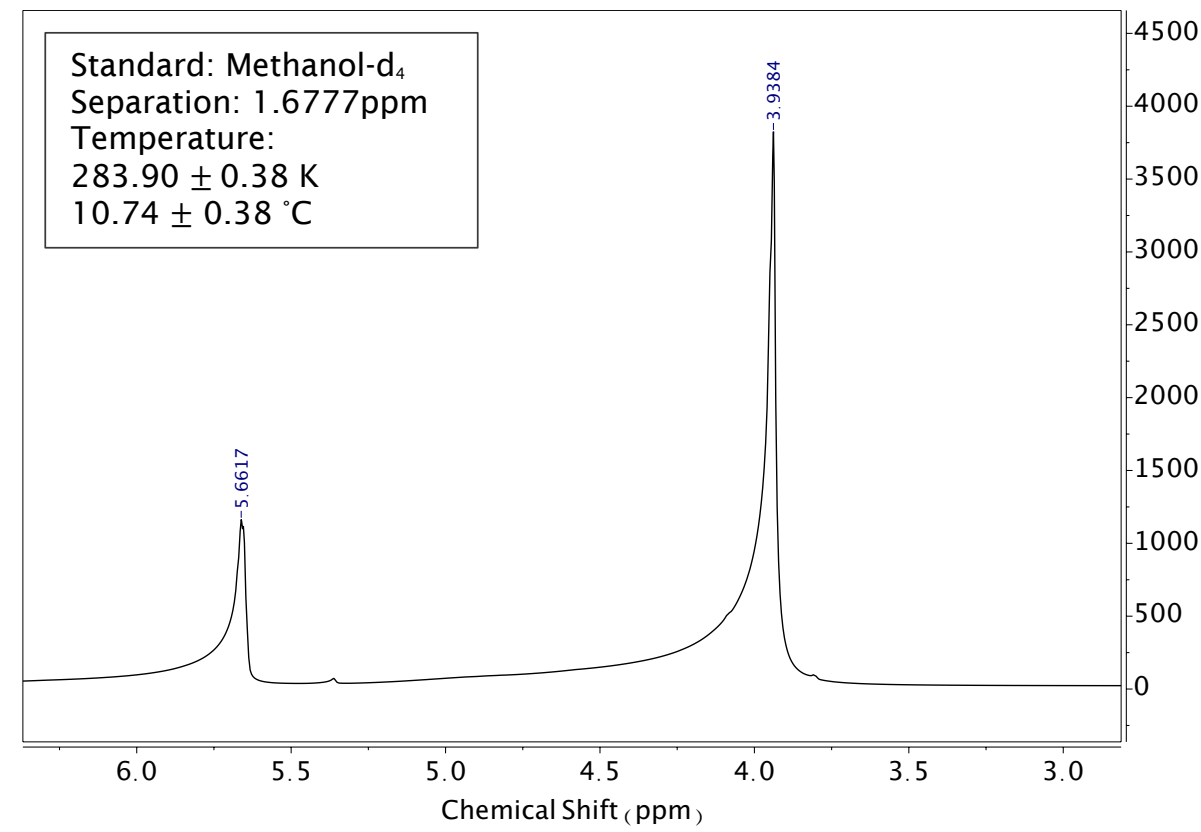

Figure S1: 1D ${ }^{1} \mathrm{H}-\mathrm{NMR}$ spectrum obtained during methanol thermometer calibration using a $500 \mathrm{MHz}$ Agilent Inova spectrometer operating at $11.75 \mathrm{~T}$. Spectra were analyzed in MNOVA without smoothing functions. Peak picking was used to measure spectral distance and determine resultant temperature reading indicated in top left-hand insert. To preserve the exact distance between peaks, phasing adjustments were not used in processing. ${ }^{1}$ 
It was critical to measure the temperature in situ using the methanol thermometer as several of the temperatures measured differed from intended settings. Specifically, measurements at $31,-9$, and $-21{ }^{\circ} \mathrm{C}$ were intended to be $30,-10$, and $-20{ }^{\circ} \mathrm{C}$. In addition, although the samples equilibrate quickly (see information about dynamic light scattering) we allowed the system to equilibrate for a full $30 \mathrm{~min}$ to ensure that the NMR probe fully equilibrated to desired temperatures before methanol thermometer and subsequent EXSY NMR experiments were performed.

\section{Differential scanning calorimetry:}

To explore the possibility for a phase transition occurring between the high and low temperature regimes, we measured our samples using differential scanning calorimetry. The data shown in Figure S2 confirm that no freezing or other major phase transition occurs over the temperature range of our EXSY NMR measurements.

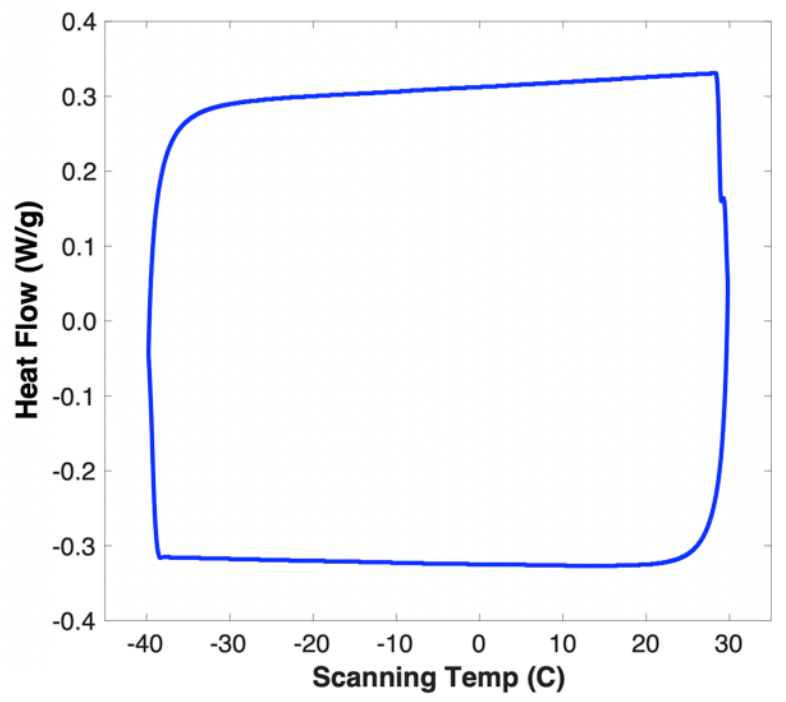

Figure S2: Differential scanning calorimetry thermogram of water/AOT/isooctane reverse micelles, $w_{0}=10$ reverse micelles loaded with a glucose:water ratio of 1:30 over the temperature range probed in the EXSY NMR experiments. The thermogram shows no evidence of a thermodynamic phase change. Initially, we scanned from high temperatures to low temperatures at a rate of $10{ }^{\circ} \mathrm{C} / \mathrm{min}$, then cycled back to room temperature by ramping up temperature from the subzero regime, to account for hysteresis effects when ramping the temperature. Measurements were repeated in triplicate. 


\section{Deuterium NMR studies:}

As described in the article, we attempted to measure the kinetic isotope effect predicted to impact $\mathrm{H}$ atom tunneling in the reported low temperature regime. As Figure S3 shows, the significantly reduced sensitivity and resolution arising from the ${ }^{2} \mathrm{H}$ nucleus parameters precluded confirmation of the kinetic isotope effect through ${ }^{2} \mathrm{H}-\mathrm{NMR}$ spectroscopy. The fast quadrupole relaxation due to the large quadrupole moment $\left(2.86 \times 10^{3}\right.$ barns $)$ of the ${ }^{2} \mathrm{H}$ nucleus makes ${ }^{2} \mathrm{H}-\mathrm{NMR}$ spectroscopy challenging in the best circumstances. ${ }^{58-60}$ The large quadrupole moment experiences significant interaction with the surrounding electric field gradients generated by both the solvent and the solute molecules, therefore making relaxation extremely efficient and broadening the signal lineshapes. ${ }^{57}$

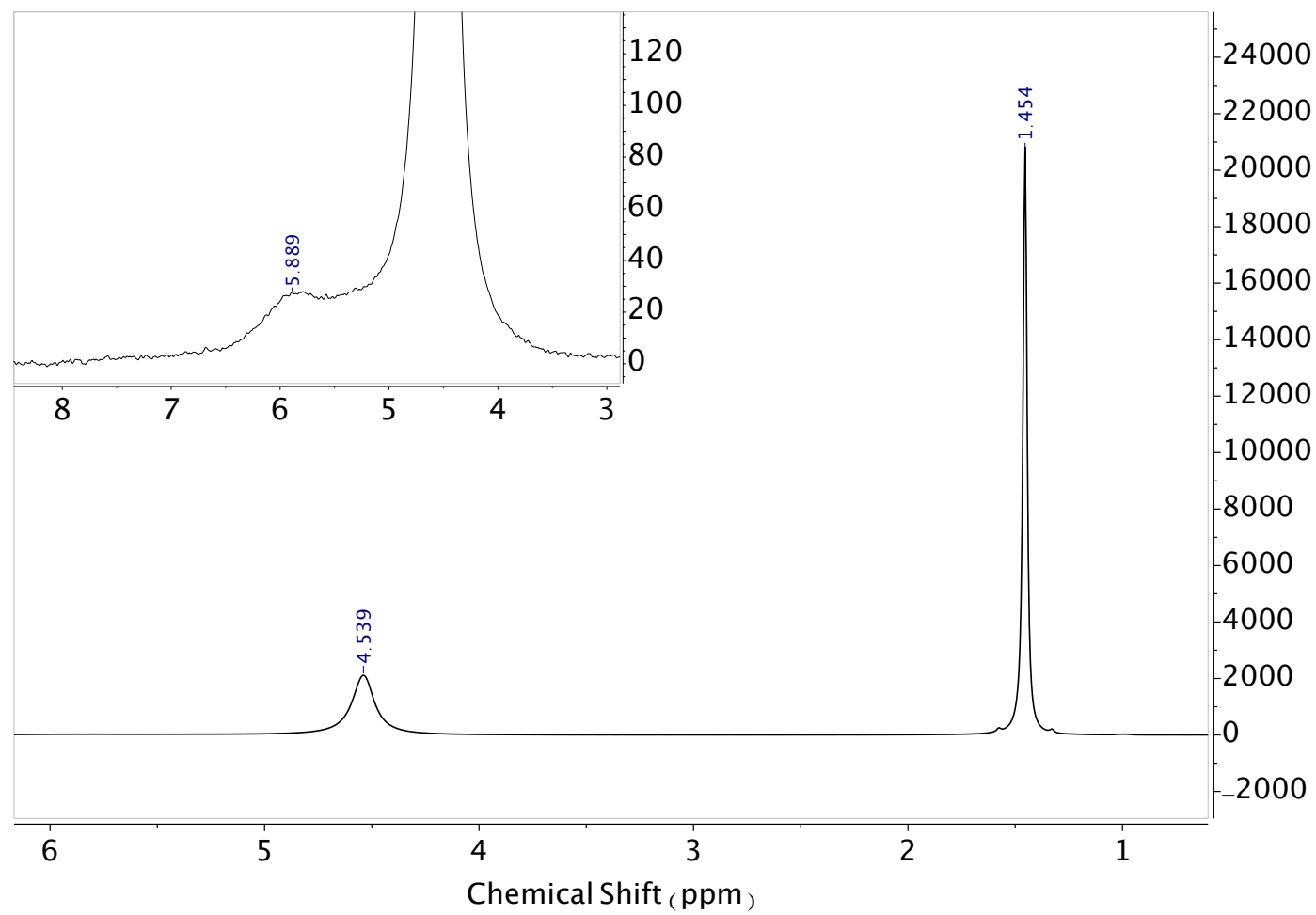

Figure S3. 1D ${ }^{2} \mathrm{H}-\mathrm{NMR}$ spectra of OD glucose loaded AOT reverse micelles.

\section{Dynamic light scattering measurements.}

Figure S4 shows size data measured for the glucose-loaded AOT reverse micelles at three different temperatures. Unfortunately, the lower limit of the temperature range for the dynamic 
light scattering (DLS) instrument employed is $275 \mathrm{~K}$ precluding size measurements over the entire temperature range probed for our EXSY NMR experiments. However, the lowest temperature we measured, $275 \mathrm{~K}$, falls within what we consider the low temperature regime for the Arrhenius plots. Should a noticeable size change effect the phase of the water core at the temperature threshold we calculate, our DLS measurements could detect such a difference at $\sim 285 \mathrm{~K}$.

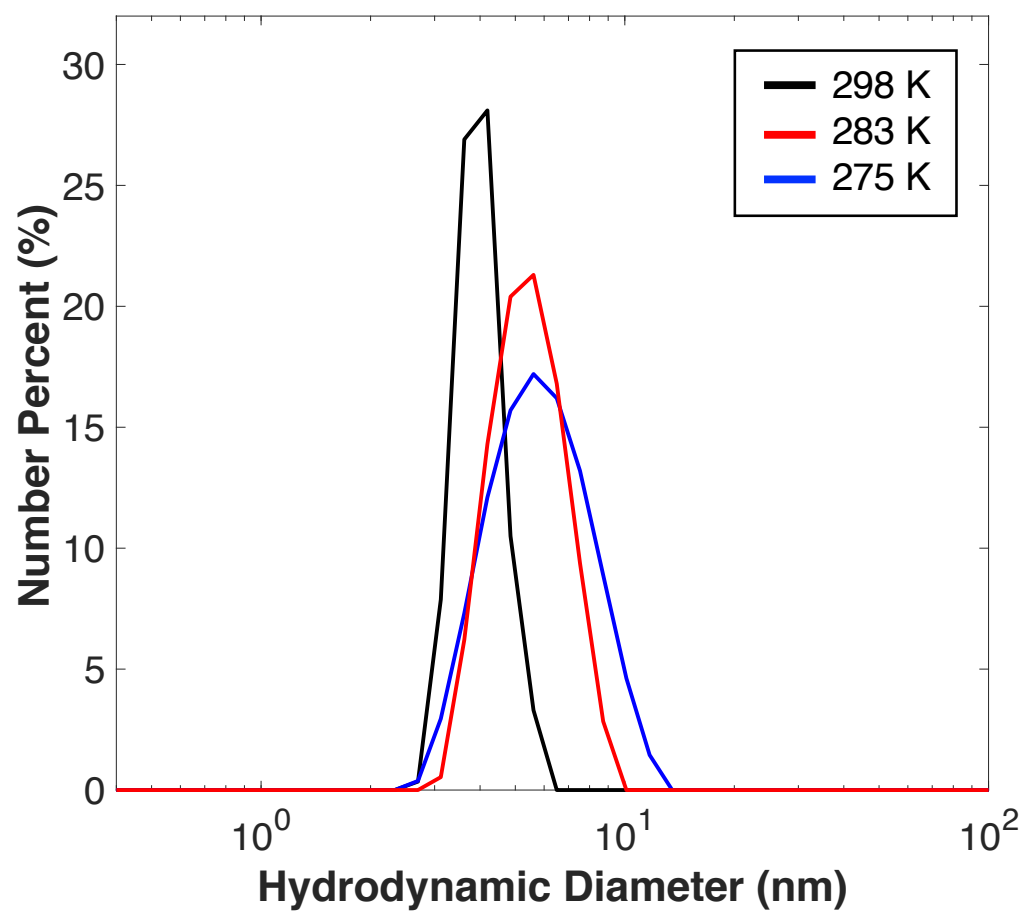

Figure S4: Particle number distribution vs. hydrodynamic diameter plots obtained from the DLS experiment for $w_{0}=10$ reverse micelles loaded with glucose at a 1:30 glucose to water ratio. The DLS data reveal that the reverse micelles are approximately $6.0 \mathrm{~nm}$ in diameter, which is consistent with our expectations for glucose loaded $w_{0}=10$ reverse micelles. The curve shown here represents an average of three sets of ten scans. The full width at half maximum of the distribution curve indicates that sample is not perfectly monodisperse; A monodisperse sample would yield a narrower intensity distribution curve centered perfectly about a single value. 
Table S1: $k_{1}$ and $k_{2}$ rate constants and associated uncertainties for chemical exchange and subsequent spin-lattice relaxation of glucose hydroxyl groups (eqn 2 and 3). Values represent the average of three independent measurements. Because we cannot unequivocally assign the peaks to the specific position, peaks 3,4 down and 3,4 up refer to hydroxyl groups associated combined 3 and 4 position on glucose.

\begin{tabular}{|c|c|c|c|c|}
\hline \multirow{2}{*}{ Temp (K) } & \multicolumn{2}{|c|}{$\alpha$-D-glucose } & \multicolumn{2}{c|}{$\beta$-D-glucose } \\
\cline { 2 - 5 } & $\mathrm{k}_{1}\left(\mathrm{~s}^{-1}\right)$ & $\mathrm{k}_{2}\left(\mathrm{~s}^{-1}\right)$ & $\mathrm{k}_{1}\left(\mathrm{~s}^{-1}\right)$ & $\mathrm{k}_{2}\left(\mathrm{~s}^{-1}\right)$ \\
\hline 304.15 & $85.7( \pm 28.2)$ & $1.2( \pm 0.3)$ & $125.3( \pm 26.3)$ & $1.2( \pm 0.3)$ \\
\hline 298.15 & $51.1( \pm 10.2)$ & $1.2( \pm 0.3)$ & $89.5( \pm 19.6)$ & $1.3( \pm 0.4)$ \\
\hline 288.15 & $14.7( \pm 3.5)$ & $1.8( \pm 0.4)$ & $23.9( \pm 8.8)$ & $1.9( \pm 0.3)$ \\
\hline 283.15 & $11.1( \pm 3.9)$ & $1.9( \pm 0.3)$ & $14.8( \pm 3.1)$ & $1.9( \pm 0.4)$ \\
\hline 273.15 & $8.9( \pm 3.0)$ & $1.9( \pm 0.3)$ & $13.9( \pm 3.3)$ & $1.9( \pm 0.4)$ \\
\hline 268.15 & $8.1( \pm 2.2)$ & $1.9( \pm 0.3)$ & $7.5( \pm 3.0)$ & $2.1( \pm 0.6)$ \\
\hline 264.15 & $6.8( \pm 1.3)$ & $1.8( \pm 0.4)$ & $5.9( \pm 1.1)$ & $2.2( \pm 0.5)$ \\
\hline 258.15 & $7.2( \pm 1.7)$ & $1.9( \pm 0.3)$ & $5.8( \pm 0.7)$ & $2.4( \pm 0.6)$ \\
\hline 252.15 & $5.5( \pm 1.5)$ & $2.7( \pm 0.7)$ & $5.5( \pm 0.7)$ & $2.3( \pm 0.5)$ \\
\hline
\end{tabular}

Table S2: Reported $\mathrm{R}^{2}$ values obtained after linearly fitting Arrhenius plots.

\begin{tabular}{|c|c|}
\hline \multicolumn{2}{|c|}{ Single Linear Fit } \\
\hline Single Linear Fit ( $\alpha$-glucose) & 0.820 \\
\hline Single Linear Fit ( $\beta$-glucose) & 0.889 \\
\hline \multicolumn{2}{|c|}{ Two Part Linear Fit ( $\alpha$-glucose) } \\
\hline High temperature Regime & 0.986 \\
\hline Low temperature Regime & 0.863 \\
\hline Two Part Linear Fit ( $\beta$-glucose) \\
\hline High temperature Regime & 0.990 \\
\hline Low temperature Regime & 0.875 \\
\hline
\end{tabular}


Table S3: Various size distributions of $w_{0}=10$ reverse micelles loaded with glucose at a 1 to 30 glucose to water ratio at different temperatures. Plot demonstrates that size of reverse micelles is temperature dependent. Average size increases as temperature increases. This suggests that water forms the stiff hydrogen bonds within the reverse micelle that may lead to an expansion of the reverse micelle core. However, the "increase" in average diameter could also be due to the increase in polydispersity, as indicated by the greater value for FWHM.

\begin{tabular}{|c|c|}
\hline $\begin{array}{c}\text { Temp } \\
(\mathbf{K})\end{array}$ & $\begin{array}{c}\text { Hydrodynamic } \\
\text { Diameter }(\mathbf{n m})\end{array}$ \\
\hline 303 & 4.53 \\
\hline 298 & 4.73 \\
\hline 293 & 5.41 \\
\hline 288 & 5.43 \\
\hline 283 & 6.11 \\
\hline 275 & 7.24 \\
\hline
\end{tabular}

Table S4: Temperature dependent width of the water NMR signal. The measured width is the result of the three separate measurements given by MNOVA during processing and analysis. $\mathrm{T}_{2}$ was calculated from the well-known formula:

$$
\Delta v=\frac{1}{\pi T_{2}}
$$

\begin{tabular}{|c|c|c|c|}
\hline $\begin{array}{c}\text { Temp } \\
(\mathbf{K})\end{array}$ & $\begin{array}{c}\text { Width } \\
(\mathbf{H z})\end{array}$ & $\begin{array}{c}\text { Std. Dev } \\
(\mathbf{H z})\end{array}$ & $\mathbf{T}_{\mathbf{2}}(\mathbf{s})$ \\
\hline 303 & 4.97 & 0.047 & 0.064 \\
\hline 298 & 5.03 & 0.047 & 0.063 \\
\hline 288 & 5.03 & 0.047 & 0.063 \\
\hline 283 & 5.61 & 0.092 & 0.057 \\
\hline 273 & 7.53 & 0.021 & 0.042 \\
\hline 268 & 7.89 & 0.082 & 0.040 \\
\hline 264 & 8.27 & 0.125 & 0.039 \\
\hline 258 & 10.54 & 0.059 & 0.030 \\
\hline 252 & 13.72 & 0.074 & 0.023 \\
\hline
\end{tabular}




\section{Additional Citations:}

(1) Van Geet, A. L. Calibration of the Methanol and Glycol Nuclear Magnetic Resonance Thermometers with a Static Thermistor Probe. Anal. Chem. 1968, 40, 2227-2229.

(2) Marshall, A. G. Calculation of NMR Relaxation Times for Quadrupolar Nuclei in the Presence of Chemical Exchange. J. Chem. Phys. 1970, 52, 2527-2534.

(3) Ludwig, R. NMR Relaxation Studies in Water-Alcohol Mixtures: The Water-Rich Region. Chem. Phys. 1995, 195, 329-337

(4) Schönhoff, M. NMR Methods for Studies of Organic Adsorption Layers. In Studies in Interface Science; Max Planck Institute: Potsdam, 2001.

(5) Karunakaran, C. Spin Resonance Spectroscopy: Principles and Applications, 1st ed.; Elsevier, 2018. 